\title{
Coexistence and Specialization of Pathogen Strains on Contact Networks
}

\author{
Ken T. D. Eames ${ }^{1,2, *}$ and Matt J. Keeling ${ }^{1, \dagger}$
}

1. Department of Biological Sciences and Mathematics Institute, University of Warwick, Gibbet Hill Road, Coventry CV4 7AL, United Kingdom;

2. Department of Zoology, University of Cambridge, Downing Street, Cambridge CB2 3EJ, United Kingdom

Submitted August 7, 2005; Accepted April 4, 2006;

Electronically published July 10, 2006

Online enhancement: appendix.

ABSTRACT: The coexistence of different pathogen strains has implications for pathogen variability and disease control and has been explained in a number of different ways. We use contact networks, which represent interactions between individuals through which infection could be transmitted, to investigate strain coexistence. For sexually transmitted diseases the structure of contact networks has received detailed study and has been shown to be a vital determinant of the epidemiological dynamics. By using analytical pairwise models and stochastic simulations, we demonstrate that network structure also has a profound influence on the interaction between pathogen strains. In particular, when the population is serially monogamous, fully cross-reactive strains can coexist, with different strains dominating in network regions with different characteristics. Furthermore, we observe specialization of different strains in different risk groups within the network, suggesting the existence of diverging evolutionary pressures.

Keywords: strains, coexistence, evolution, network, monogamy.

The vast majority of infectious disease models consider a single host species and a single strain of pathogen. However, in recent years increasing attention has focused on the interaction of multiple strains or even different species of pathogen. Areas of research have included the interaction between different strains of influenza and the ensuing antigenic drift (Smith et al. 2004), the shifting pat-

\footnotetext{
* E-mail: k.eames@warwick.ac.uk.
}

† E-mail: m.j.keeling@warwick.ac.uk.

Am. Nat. 2006. Vol. 168, pp. 230-241. (C) 2006 by The University of Chicago. 0003-0147/2006/16802-41226\$15.00. All rights reserved. terns of respiratory syncytial virus (RSV) strains (Frabasile et al. 2003), the competition between wild-type and antibiotic-resistant strains of methicillin-resistant Staphylococcus aureus (MRSA; Baba et al. 2002), and the interference through convalescence between immunologically independent childhood diseases (Rohani et al. 2003). The competitive interaction between two strains is key to understanding disease evolution, both in terms of predicting future trends and of interpreting current patterns (Grenfell et al. 2004). Central to all these models of pathogen competition is the tenet that complete cross-immunity between strains leads to the competitive exclusion of one strain; the strain that can best exploit the pool of susceptible individuals forces other strains to extinction (May and Anderson 1983; Bremermann and Thieme 1989; Turner and Garnett 2002). Here we challenge this principle and show that cross-immune strains can coexist in a heterogeneous population with variations in the rate of turnover of contacts. These conditions, displayed in many social settings, are most clearly realized in models of sexually transmitted diseases (STDs) where the population can often be partitioned into a highly active core group and a less active periphery and in which sexual contacts are necessarily sequential.

The maintenance and prevalence of STDs is strongly influenced by heterogeneities in mixing within the population. Most individuals have relatively few long-lived sexual partnerships, while a small core group has many partnerships of far shorter duration (Johnson et al. 1994; Rothenberg et al. 1996; Zenilman et al. 1999; Johnson et al. 2001). Infection is concentrated within core groups and is found at a much lower level in the general population. Like-with-like (assortative) mixing within populations separates population subgroups and enhances differences in prevalence between behavioral classes.

Contact networks, in which individuals appear as points and interactions between individuals appear as lines between points, have been used for some time as a means of understanding how the structure of a population affects processes within it, including the spread of infectious diseases (Wasserman and Faust 1994; Rothenberg et al. 1996; 
Jolly and Wylie 2002). Networks have been particularly well used in the case of STD epidemiology because sexual mixing networks are more straightforward to define and to measure than mixing networks associated with infections spread through social contact. In contrast to familiar completely mixing mean-field models (Anderson and May 1992), each individual in a network only interacts directly with a small fraction of the population.

Much of the research on sexual networks looks at snapshots (perhaps with a slow shutter speed) of contact networks, which are often interpreted as representing all current partnerships (Ferguson and Garnett 2000; Eames and Keeling 2002; Jolly and Wylie 2002; Bearman et al. 2004). For modeling purposes, connections within these networks are assumed to be fixed and concurrent. Often a more realistic approach is to assume the population to be serially monogamous, with each individual being in at most one partnership at any one time (Dietz and Hadeler 1988; Altmann 1995; Kretzschmar and Dietz 1998; Eames and Keeling 2004). This framework includes situations in which partnerships are extremely short and in which partnerships form repeatedly between the same two individuals. Serial monogamy slows the spread of infection and reduces persistence. Furthermore, for a pathogen to be sustained in the population, partnerships must last long enough to transmit infection but must break up fast enough for an individual to remain infectious until the next partnership forms. Prevalence is maximized when partnership formation is rapid and partnership duration is intermediate (Kretzschmar and Dietz 1998; Eames and Keeling 2004).

We expect highly connected core group individuals to have many short-duration partnerships and those individuals with fewer network contacts to have fewer longer relationships (Hethcote and Yorke 1984; Johnson et al. 2001). We investigate the possibility of coexistence of pathogen strains that specialize on these different regions of the network. We demonstrate that rapidly transmitted strains are favored where partnership turnover is fast, whereas slow strains with long infectious periods dominate where partnership turnover is slow.

We use two methods to investigate the dynamics of competing strains: individual-based stochastic simulations carried out on computer-generated networks and deterministic pairwise approximations. The former approach includes all details of a population's interactions while the latter has the advantages of ease of parameterization and interpretation, as well as being considerably less computationally intensive. The pairwise model is the natural extension of standard compartmental modeling methods (Anderson and May 1992) applied to network-based interactions.

Coexistence of strains or of species has previously been attributed to coinfection, to complex infection dynamics, to spatial segregation, or to transient phenomena (Nowak and May 1994; van Baalen and Sabelis 1995; Boots and Sasaki 1999; Chesson 2000; Keeling et al. 2003; Amarasekare et al. 2004; Snyder and Chesson 2004). Here we show that none of these mechanisms is necessary and that different partnership turnover rates for high- and low-risk individuals allow two different strains to coexist within a network. Behavioral heterogeneities within a single connected population provide a range of niches in which differing strains can dominate. The result appears in both the idealized deterministic model and in detailed stochastic simulations. We discuss implications for the evolution of strains within different regions of a contact network.

\section{Methods}

Throughout, we use a simple susceptible-infected-susceptible (SIS) model of infection in which individuals are either susceptible or infectious and on recovery from infection become susceptible once again. Pathogens are described by their transmission rate across a contact, $\tau$, and their recovery/treatment rate, $g$ (giving an average infectious period of $g^{-1}$ ).

The interactions between individuals are described by a network of contacts representing all potential sexual partnerships. This underlying potential network is fixed, but the nature of the links, which may be active or inactive, is not. All individuals are assumed to be serially monogamous, that is, in an active partnership with at most one of their potential partners at any one time (fig. 1). We assume that the number of potential partners (termed the neighborhood size) in the network provides a measure of an individual's sexual activity and hence that the rate of partnership breakup depends on the size of the potential neighborhood; the more potential partners an individual has, the shorter each of his partnerships will be. Highly connected individuals correspond to a core group with many short-duration partnerships (Rothenberg et al. 1996; Zenilman et al. 1999). Throughout, we assume that inactive links between unpartnered individuals become active at a constant rate, $\alpha$, and that partnerships between two actively connected individuals with potential neighborhood sizes $n$ and $m$ break up at rate $\rho \times(n+m)$; this can be seen as each individual within a partnership initiating breakup at a rate proportional to their neighborhood size.

We model the interaction between two strains by allowing an individual to be infected with at most one strain at a time, recovery from which gives no immunity to either strain (Turner and Garnett 2002). Each strain interferes with the other by reducing the pool of susceptible individuals. The competition introduced by this interference 


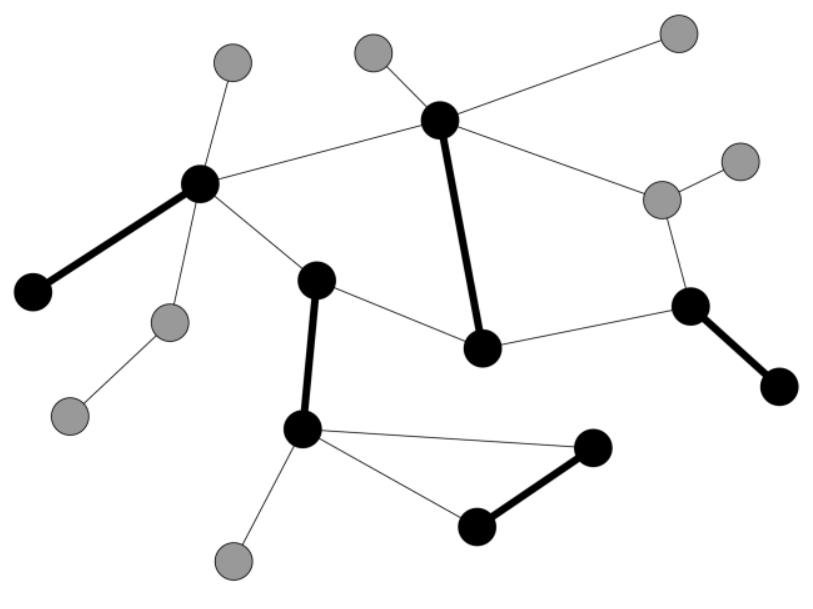

Figure 1: Illustration of partnerships on a potential network. Individuals are represented by circles and an individual's set of potential partners by links to other circles. Only one active partnership per individual is permitted at any time; active individuals (those in a partnership) and links are shown in black. Over time, active links may become inactive, allowing individuals to form connections elsewhere in their potential neighborhood.

would lead us to expect, in a homogeneous population, that the strain best able to exploit the population would competitively exclude the other (May and Anderson 1983; Bremermann and Thieme 1989; Turner and Garnett 2002). In epidemiological terms, the reproductive ability of a pathogen is summarized by the quantity $R_{0}$, the basic reproductive ratio, defined as the number of secondary cases generated by a single infectious individual in an entirely susceptible population.

Two different approaches are used to model the epidemic. The first is an individual-based stochastic model of the population that explicitly includes all interactions within the network. The second is a deterministic pairwise approach designed to capture the network spread of infection without requiring all the details of the population's behavior for its parameterization. These approaches complement each other, with the network model giving a detailed stochastic picture of the entire system and the pairwise approach enabling definitive results to be obtained relatively rapidly over a wide range of parameters.

Both cases require the numerical simulation of epidemics; although the pairwise approach provides a simplification of the network-based epidemic, it is nevertheless intractable to pen-and-paper methods. Even when considering a single strain in a homogeneous population, the inclusion of both partnership and infection dynamics makes the system too complex for direct solution. Nevertheless, the deterministic nature of the model means that the stability of coexisting strains is straightforward to assess.

\section{Network Model}

To investigate epidemic spread on heterogeneous networks, we modify previous network-generating techniques (Eames and Keeling 2002; Read and Keeling 2003) in order to divide the population into two behavioral subgroups. The approach taken here is one of several that could be used; the aim was to generate distinguishable population subgroups via a relatively minor and straightforward adaptation of previous methods.

Individuals are distributed in a square in two-dimensional space. Ten percent of the population is assigned to the core group, and each individual is allocated a desired number of potential partners, those in the core group averaging approximately 20 and those in the rest of the population averaging approximately four. Core group individuals are positioned in the central $1 \%$ of the square, while the rest of the population is evenly distributed throughout (thus core group individuals are more densely packed). Connections are formed preferentially between nearby individuals, ensuring that the majority of the connections individuals make are with similar individuals. A Gaussian connection kernel is used, parameterized to give the desired distributions of partnerships; here, over $90 \%$ of connections are within group, giving an assortative pattern of mixing.

When running epidemics on these networks, we seed the epidemic by infecting a randomly selected $1 \%$ of the population. This relatively large fraction (compared with only infecting a single individual) reduces the chance of extinctions caused by small stochastic fluctuations. When two strains are examined within the same network, $1 \%$ of the population is infected with each strain. The state of the network (including both its infection and partnership properties) is updated event-by-event using the direct Gillespie algorithm (Gillespie 1977; Gibson and Bruck 2000).

\section{Pairwise Model}

Complete network models have several advantages: they include all interactions within a population, they track the infection status of each individual, and, being stochastic, they can capture some of the intrinsic variation within the system. However, these advantages can also lead to problems in terms of interpretation, parameterization, and computational time. The number of possible events increases rapidly with population size, and the stochastic nature of the epidemic means that multiple runs are required to determine model behavior with confidence, which limits the size of population for which such an 
approach is feasible. The requirement that every interaction within the population is known, while satisfied for the artificially generated networks used here, is impractical when applied to any real-world population: it is seldom, if ever, possible to document all the interactions between individuals, and therefore networks can only be approximated at best.

The recognition of these problems has motivated the development of modeling techniques that can be used to represent network processes. The more familiar mean-field approaches (Anderson and May 1992) are not appropriate because they allow all individuals in the population to interact weakly with each other; while this may make sense for infections spread through casual social contact, in which many interactions take place with many people, it is an unreasonable assumption when the interactions within the population change slowly relative to the timescales of the epidemic. In the models discussed here, the underlying potential network is fixed, and therefore each individual can only ever come into direct contact with a small fraction of the population, a constraint also applicable to the real world of sexual relationships.

Pairwise models have been developed as a compromise between standard mean-field and full-network models (Keeling et al. 1997; Rand 1999; Ferguson and Garnett 2000); recognizing that the infection status of interacting individuals is likely to be correlated, pairwise models treat connected pairs of individuals as their basic variables. The difference between mean-field and pairwise models is seen in the way they model the change in the number of infectious individuals, $[I]$. A mean-field model would take the form

$$
[\dot{I}]=\beta[S] \times[I]-g[I],
$$

where $[S]$ is the number of susceptible individuals and $\beta$ is a compound parameter containing details of transmission rate and mixing behavior. In contrast, the pairwise model uses

$$
[\dot{I}]=\tau[S I]-g[I]
$$

where $\tau$ is the transmission rate per contact and $[S I]$ is the number of susceptible-infected pairs within the network. Thus, the pairwise model explicitly includes interactions between susceptible and infectious individuals. Rather than having only $[S]$ and $[I]$ as variables, the pairwise approach models the dynamics of all pair types (such as $[S S],[S I]$, and $[I I]$ ) during the epidemic.

The serially monogamous interactions considered in this article require a further adaptation: individuals and links within the potential network can be either active or in- active, and infection can only pass between network neighbors if the link between them is active; we use $\leftrightarrow$ to denote active links and the subscript $A$ to denote active individuals (i.e., those involved in an active link), hence

$$
[\dot{I}]=\tau\left[S_{\mathrm{A}} \leftrightarrow I_{\mathrm{A}}\right]-g[I] .
$$

The model must keep track of the four processes taking place within the potential network: transmission of infection, recovery, partnership formation, and partnership breakup. By considering how the numbers of different types of pairs within the network change as the epidemic progresses, a set of differential equations describing the epidemic can be derived. These equations and some more details of the pairwise approach can be found in the appendix in the online edition of the American Naturalist.

It is straightforward to adapt the pairwise model to represent a heterogeneous population and to consider multiple competing strains of infection (appendix). The model can easily be parameterized to investigate an epidemic on any network of interest and is straightforward to interpret. It allows rapid calculation of $R_{0}$ from the initial growth rate of the epidemic (Eames and Keeling 2002), which can be used to assess the outcome of strain competition. The model has further advantages in terms of speed of simulation.

\section{Coexistence}

Standard theory for randomly mixing populations suggests that the strain with the greatest $R_{0}$ will dominate and force the other strains to extinction (May and Anderson 1983; Bremermann and Thieme 1989; Turner and Garnett 2002). This property continues to hold in a polygamous network, in which all connections are capable of transmitting infection at all times (Eames and Keeling 2002), and a single strain dominates irrespective of network heterogeneities.

In a serially monogamous population the situation is more complex. When the network is homogeneous, only the strain with the largest value of $R_{0}$ will persist, but the identity of this strain may depend on the properties of the network. Figure 2 shows $R_{0}$ in a homogeneous network, calculated using the pairwise model for a single strain (Eames and Keeling 2002); $R_{0}$ depends both on the potential neighborhood size and partnership breakup rate. The breakup rate that maximizes $R_{0}$ depends on the properties of the network in which the infection exists, suggesting that the precise identity of the dominant strain may depend on the attributes of the host population.

Figure $3 A, 3 B$ shows the long-term distribution of two strains in an assortative, heterogeneous, monogamous potential network. In figure $3 A, 3 B$ the same stochastically 


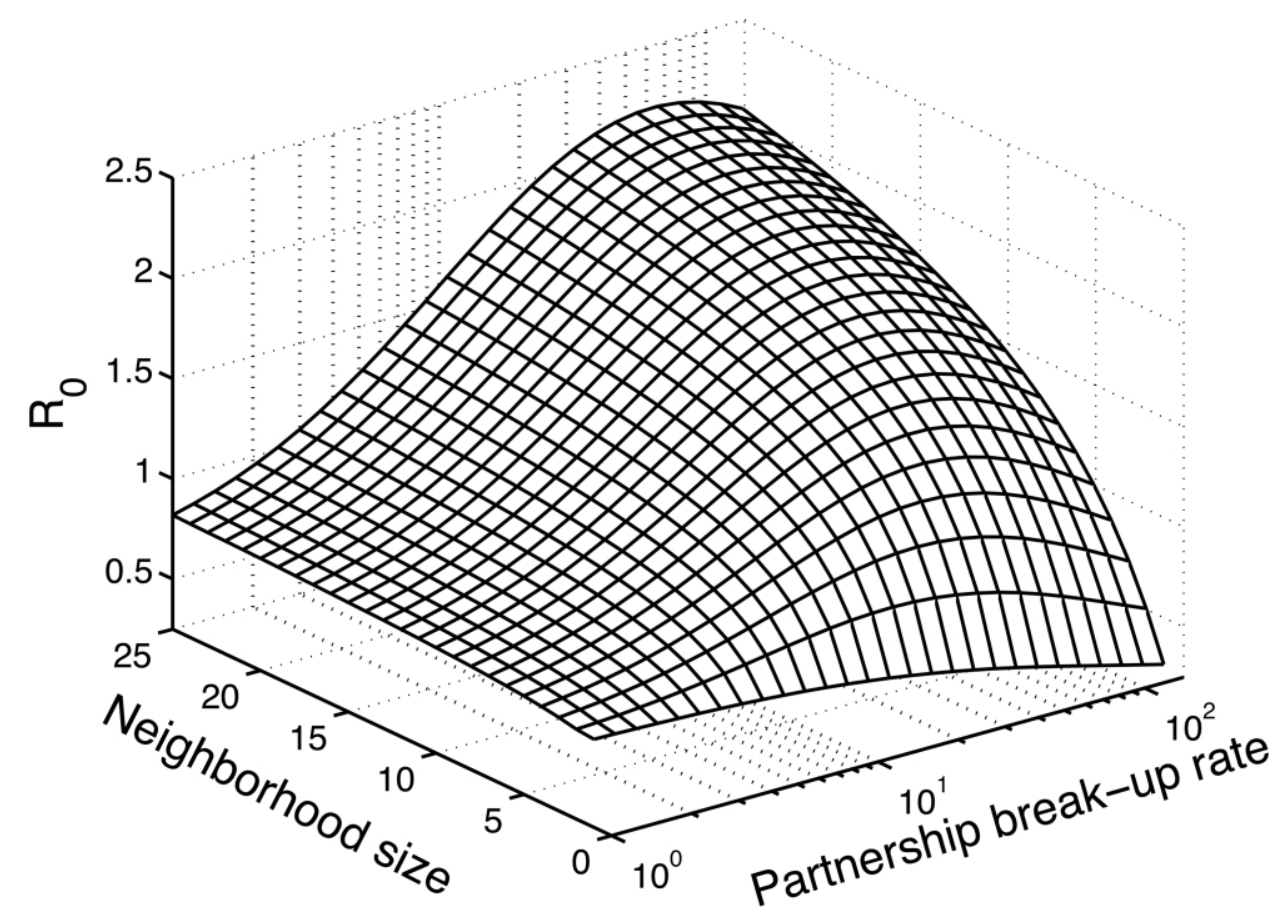

Figure 2: Basic reproductive ratio, $R_{0}$, as a function of potential neighborhood size and the partnership breakup rate on a homogeneous potential network. Throughout, $R_{0}$ is calculated from the growth rate of the early stage of the epidemic (Keeling et al. 1997).

generated network of 5,000 individuals is used: in figure $3 A$ it is used to parameterize the pairwise model, and in figure $3 B$ it is used as the setting for the stochastic model. The population is broken down by neighborhood size to show how different strains dominate in different regions of the network. In a mean-field or polygamous system, only one strain would persist (in this case the high transmission rate, short infectious period strain would dominate as it happens to have the larger $R_{0}$ for the parameters used). In contrast, in both a deterministic (fig. 3A) and a stochastic (fig. $3 B$ ) monogamous population, this strain persists less well in the low-risk group, where partner change is slow because infected individuals tend to recover before changing partner. Thus, when there is a core group and a low-risk group, the two strains coexist, one dominating in each group. Although both models are carried out on the same network, the difference in strain distribution is less pronounced in the pairwise than in the stochastic model because the pairwise model, which considers no paths within the network of length more than two, cannot fully represent the separation of individuals seen in the complete network.

In figure $3 C, 3 D$, the properties of strain 1 are fixed (at $\tau=8, g=2$ ) and a range of values for strain 2 is explored

Figure 3: Coexistence of two cross-resistant strains in serially monogamous networks. $A$, The equilibrium distribution of infection predicted by the pairwise model, as parameterized by a heterogeneous network of 5,000 individuals. The numbers of individuals of each neighborhood size are shown as bars: $90 \%$ of the population is in the low-risk group (neighborhood size $\leq 10$ ) while $10 \%$ is in the core group with larger neighborhood size. The division of infection between the two strains throughout the population is shown; the slow strain $(\tau=8, g=2$; red $)$ dominates prevalence in the low-risk region while the fast strain $(\tau=30.5, g=6$; blue $)$ dominates in the core group. $B$, Stochastic simulations give similar results on the same network of 5,000 individuals. The fraction of infection due to each strain is again shown in red $(\tau=8, g=2)$ and blue $(\tau=30.5, g=6)$. Points show the amount of variation from 1,000 stochastic runs with $90 \%$ error bars plotted for the two representative types (four and 20 neighbors). $C$, The coexistence region for a network with just two types of individual: high risk ( 20 neighbors, $10 \%$ of the population) and low risk (four neighbors), as predicted by the pairwise model. Strain 1 has its parameters fixed $(\tau=8, g=2$; dot), while those of strain 2 are varied; the blue and red curves show where the identity of the dominant strain changes in high-risk and low-risk groups, respectively. When each high-risk individual has two contacts in the low-risk group, the coexistence regime shrinks to the gray shaded region. $D$, Stochastic iterations of infection give similar results: average coexistence times (up to a maximum of 100 years) are shown as the parameters of strain 2 vary, on a network of 5,000 individuals. Throughout, $\alpha=20$ and $\rho=5$; all rates are events per year. 
응 웅요 웅 으 (sıеәK) uo!̣eınp әวuәเs!xวоว
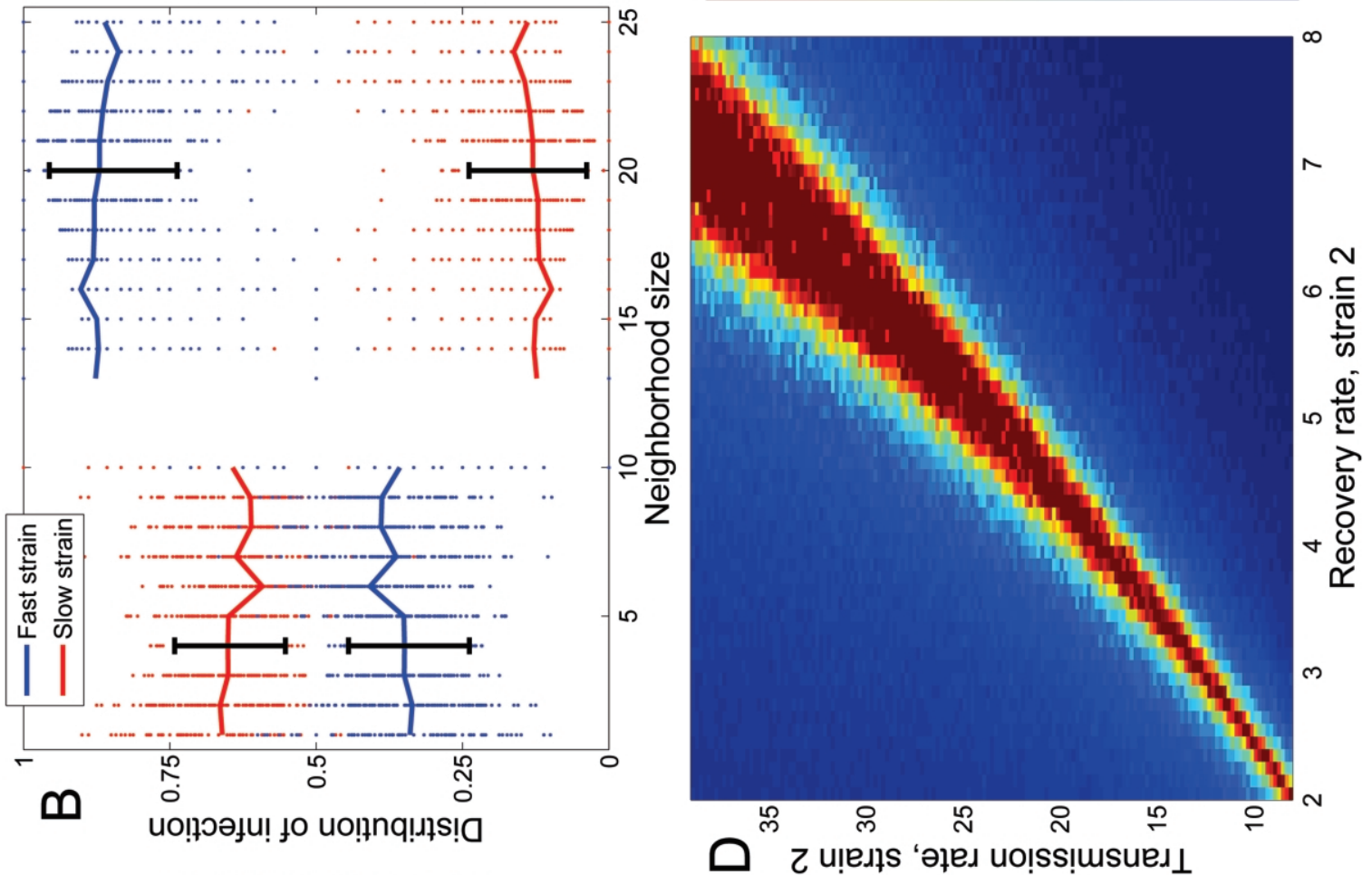

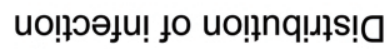

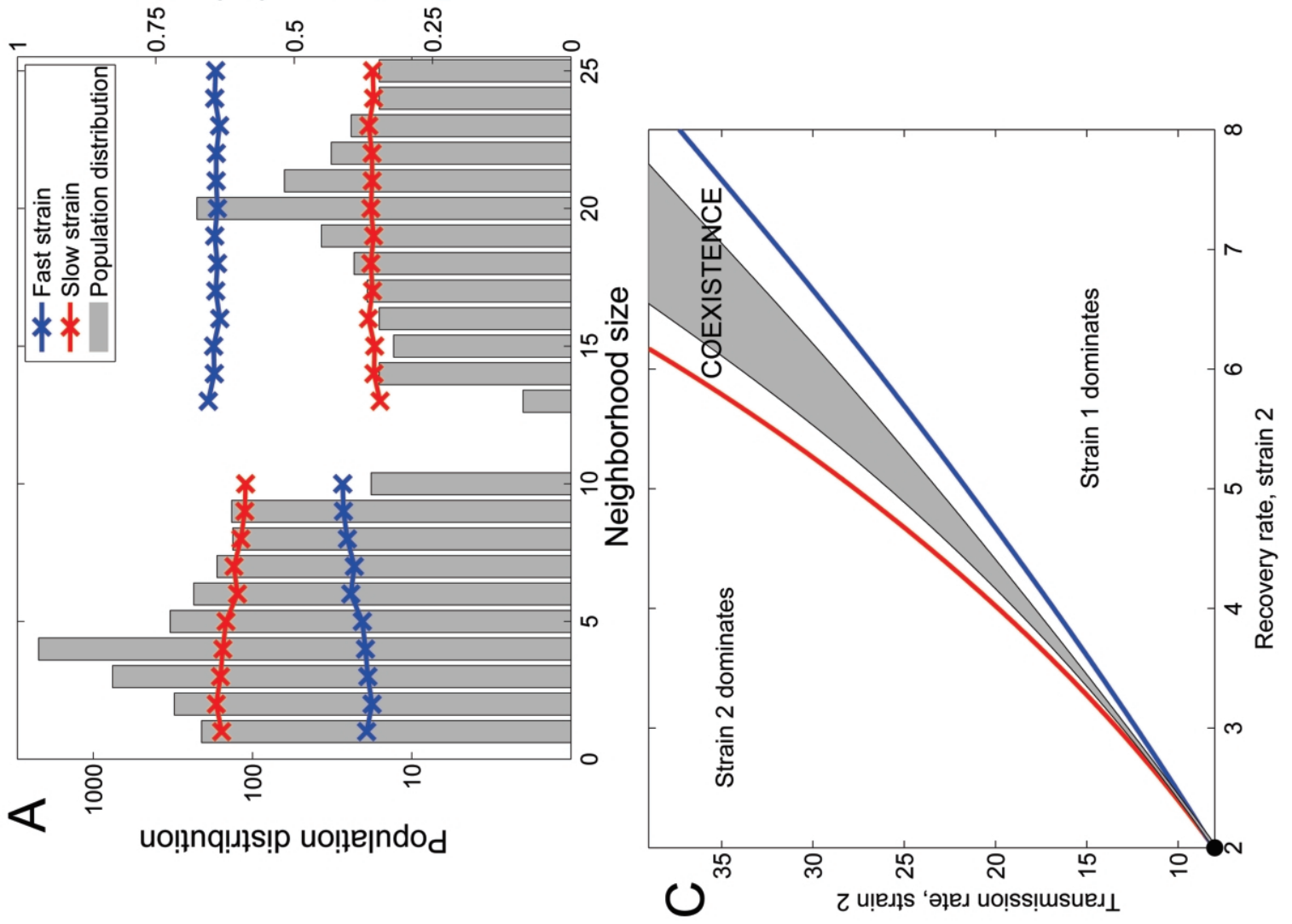


with the network structure held constant. The figures show the outcome of competition between the two strains in a simple heterogeneous network consisting of a core group (20 neighbors) and a low-risk group (four neighbors). The colored lines in figure $3 C$ separate regions of different equilibrium behavior, as predicted by the pairwise model. In the region between the red and blue lines, coexistence is possible, with the fast strain dominating in the core group and the slow strain dominating in the low-risk group. We see that similar strains cannot easily coexist (Nowak and May 1994), while as the two strains become increasingly different, the region of coexistence widens. When there is no contact whatsoever between the highrisk and low-risk groups, any set of parameters lying between the lines will lead to strain coexistence. When there is mixing between population subgroups, coexistence is less likely (fig. 3C, gray region) — strong assortativity maximizes the potential for coexistence. Nevertheless, even when mixing is entirely disassortative (i.e., all partnerships are between individuals with different characteristics), there will be a line in parameter space on which the two strains are perfectly balanced. Coexistence in a stochastic model is shown in figure $3 \mathrm{D}$ giving broadly similar results, with coexistence being possible due to behavioral heterogeneities in the population.

Figure 4 uses the pairwise model to explore the influence of assortativity further; the range of parameters for which coexistence of strains is possible increases markedly as the interaction between population subgroups falls. However, we also see that even when most of the contacts of the core group are with the low-risk population, coexistence is still possible, albeit only in a narrow region of parameter space. Thus, although assortative mixing promotes strain coexistence, it is not an absolute requirement. In a potential network in which the connections are formed at random, there is still sufficient contact between similar individuals for two strains to persist-random mixing permits coexistence in a heterogeneous population. We note that it is possible in theory for a limitless number of strains to coexist, provided that the network of interactions consists of sufficiently different and well-defined risk groups.

\section{Specialization}

Owing to the complex relationship between $R_{0}$ and the partnership turnover and infection parameters, different risk groups experience different evolutionary pressures. Thus, while within each group there is always evolutionary pressure toward larger values of $R_{0}$, this pressure drives phenotypes in differing groups toward different combinations of infectious period and transmissibility. Hence, the same initial infection evolving in a heterogeneous environment will generate diverging strains. The direction of maximal evolutionary pressure, and therefore the most likely evolutionary path, differs between high- and lowrisk groups. Where partnership turnover is rapid, higher values of the transmission parameter are selected, whereas where partnership turnover is slow, selection favors a long infectious period.

Unconstrained evolution will eventually lead to strains with both high transmission rates and long infectious periods irrespective of the risk groups involved. However, if, as suggested, a trade-off between transmission and infectious period exists (Bremermann and Thieme 1989; Frank 1996; Boots and Sasaki 1999; van Ballegooijen and Boerlijst 2004) such that diseases are constrained to lie within certain regions of parameter space, then evolutionary pressures can lead to the divergence of strains and ultimately to the long-term coexistence of different strains specialized within particular risk groups. To investigate this scenario, we assume a trade-off between recovery/treatment rate, $g$, and transmission rate, $\tau$, of the form $g=a+b \tau^{c}$, with the constraints that $a, b>0$ and $c>1$ (van Baalen 2002): under this assumption, more easily transmitted strains are shorter lasting, and an enhanced immune response or more vigorous treatment efforts lead to a nonlinear relationship. Figure $5 \mathrm{~A}$ shows how $R_{0}$, calculated using the pairwise model, depends on the transmission rate under this assumption. When $\tau$ is low, the small transmission probability generates a low value of $R_{0}$, whereas large $\tau$ leads to a short infectious period, which again reduces $R_{0}$. At some intermediate value, $R_{0}$ is maximized. However, in contrast to standard models, which predict that $R_{0}$ is maximized when $\tau / g$ is maximized (Bremermann and Thieme 1989; van Baalen 2002), the monogamous model predicts that the position of this maximum depends on the network characteristics of the host population, as suggested by figure 2 . As expected, the low-risk group favors slow strains (fig. 5, circle) whereas fast strains are more successful when mixing is rapid (fig. 5, triangle).

Figure $5 A$ also allows us to calculate the region in parameter space where coexistence of strains is possible; for instance, if one strain maximizes its $R_{0}$ in the low-risk group (circle), a range of other strains (thick black curve) will outcompete it in the core group, thus allowing two strains to persist in the population.

The precise location of the coexisting evolutionary optima depends on the shape of the trade-off assumed. The lower the sensitivity of $g$ is to changes in $\tau$ (i.e., when $a$ is large or $c$ small), the greater the distance between optimal strains in the different network regions (fig. $5 B$ ). Clearly, the degree to which coexisting strains differ will depend on the trade-offs in the system, but in all cases, the same general result holds true: fast strains are favored where partnership dynamics are fast. 


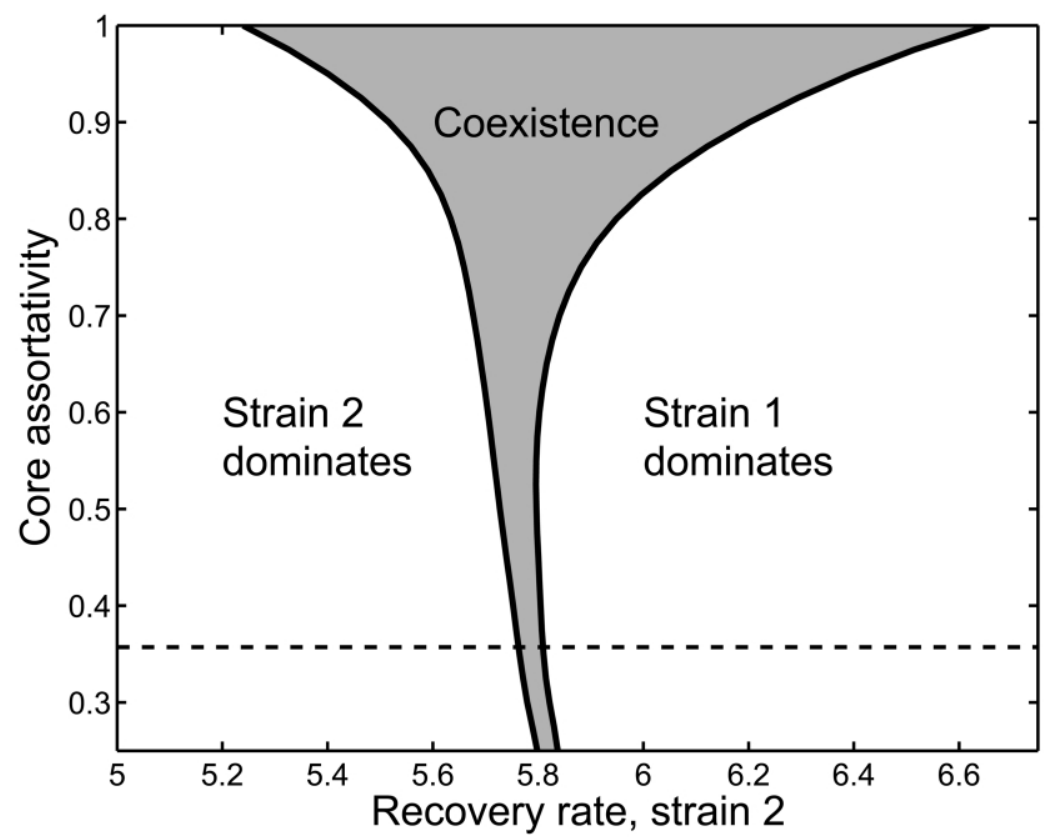

Figure 4: Effect of assortativity on coexistence in a population with two behavioral subgroups as in figure $3 C$, as predicted by the pairwise model. The coexistence region is plotted for a second strain with a fixed value of transmission rate $(\tau=30)$ for a range of population mixing patterns. Core assortativity is here defined as the proportion of potential partners of the core group that themselves lie within the core group; thus, when core assortativity $=1$, the two groups do not interact, but when core assortativity $=0.5$, half of the core group contacts are in the noncore group. The dashed line shows the level of assortativity corresponding to random mixing.

\section{Discussion}

We have demonstrated that the simple SIS infection process together with complete cross-immunity can give rise to coexisting strains of a pathogen when the social context of infection is taken into account. It is frequently the case, particularly for STDs, that contacts between individuals leading to transmission are not instantaneous but have a finite duration, a consideration not included in standard mean-field models. This constraint means that a successful pathogen not only has to transmit during the course of a partnership but also must survive between partnerships. The rate of partner change therefore exerts different pressures in high-mixing and low-mixing populations, enabling pathogens to specialize and coexist. Coexistence thus requires behavioral diversity within the population and is enhanced by assortative mixing. These conditions are, to a reasonable approximation, the norm for sexual partnerships; even within the core group, "concurrent" relationships can be viewed as rapidly undertaken and repeated monogamous partnerships.

The stable, deterministic, coexistence observed is derived from the presence of finite-length interactions, and hence it is no surprise that simpler pair formation models (Altmann 1995; Kretzschmar and Dietz 1998) can generate similar results (not shown). This similarity is encouraging, suggesting that coexistence is not merely an artifact of the modeling approach used but is a more general phenomenon. The result arises because of different partnership durations in differing regions of the transmission network, which come about because of the assumption that individuals with more potential partners tend to form shorterlived relationships. Similar coexistence results are generated if the partnership breakup parameter itself is assumed to vary, even without the presence of different numbers of potential partners; if one group has long partnerships and one group has short partnerships, again coexistence is predicted. It is possible for any number of strains to coexist, provided that there are sufficiently many behaviorally distinct population subgroups. Practically, however, there is a very limited range of parameters within which nearby strains can coexist, suggesting that strains must be a reasonable distance apart before coexistence is likely.

The results presented here echo a general tenet in ecology that heterogeneities can promote coexistence by providing a range of ecological niches for species or pathogens (Tilman 1994; Bolker and Pacala 1999; Chesson 2000; Amarasekare et al. 2004; Snyder and Chesson 2004). In an epidemiological setting, heterogeneity of the network 

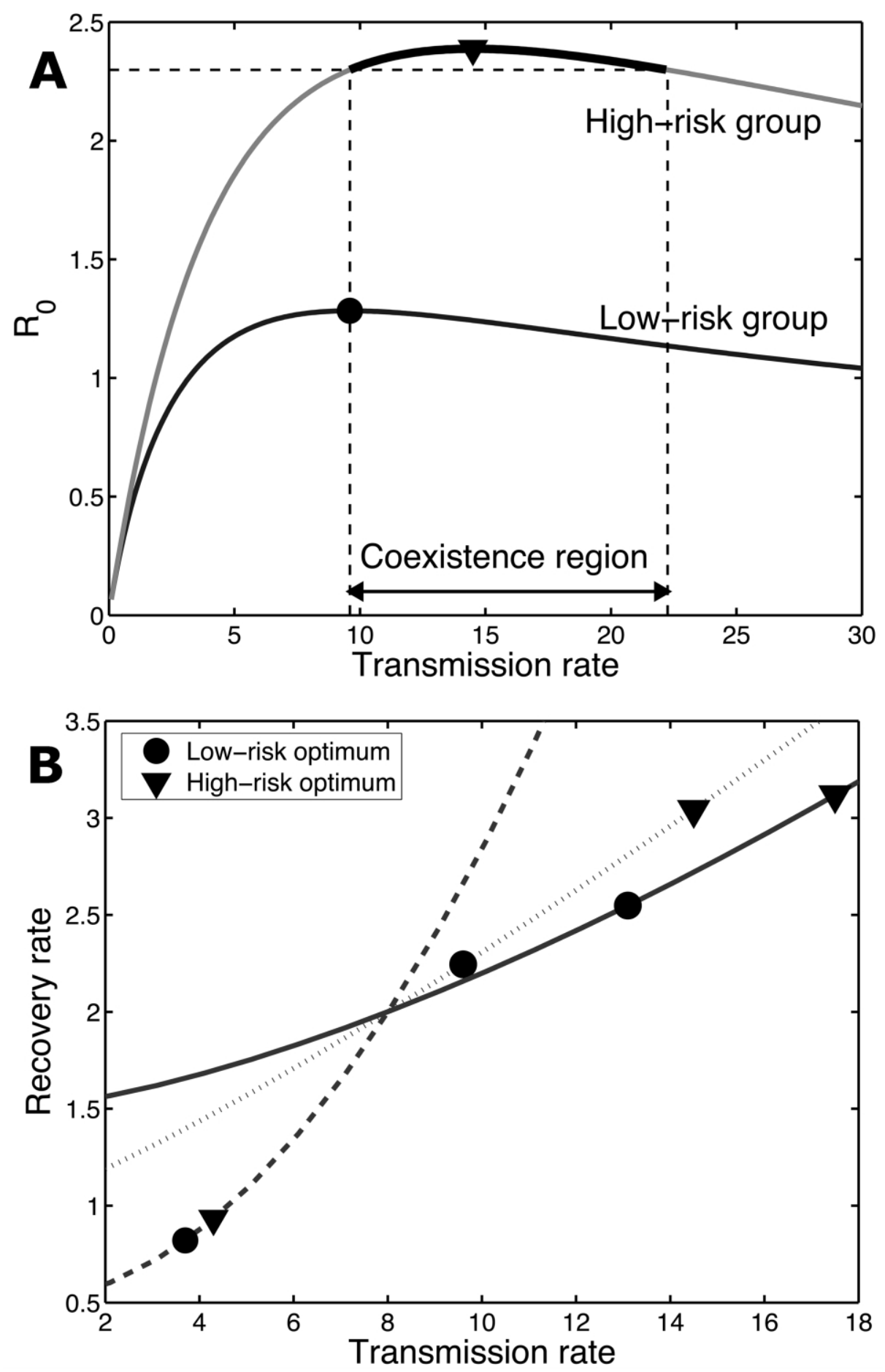

Figure 5: Effect of transmission-recovery trade-offs on strain coexistence. $A, R_{0}$ as a function of transmission rate in high-risk (upper gray line) and low-risk (lower black line) groups. The trade-off function $g=1+0.0825 \tau^{1.2}$ is used, with the value 0.0825 chosen to ensure that the parameter combination $\tau=8, g=2$ (as used in fig. 3) is permitted. The $R_{0}$ maxima are denoted by a circle and a triangle in the low- and high-risk groups, respectively. Also shown is the range of values of transmission rate for a second strain that allows it to coexist with a strain that is optimal in the low-risk group (thick black line). $B$, Evolutionarily optimal parameter values for infection in low-risk and high-risk groups for three trade-off curves of the form $g=a+b \tau^{c}$. The values $a=0.5, c=2$ (dashed line); $a=1, c=1.2$ (dotted line); and $a=1.5, c=1.5$ (solid line) are used with $b$ chosen in each case to ensure that the curve passes through $\tau=8, g=2$. 
is insufficient; the existence of different behavioral groups is not enough to allow persistence of more than one strain. However, when partnerships within a population are considered, we have shown that serial monogamy coupled with a variation in partnership turnover rates leads to different trade-offs between colonization (transmission rate) and persistence (infectious period) in different parts of the network, thus allowing several strains to coexist.

Regions of the network with different host behavioral characteristics also exert different evolutionary pressures, and this divergence promotes speciation within a population, with the strains in different behavioral subgroups moving farther apart. The role this divergence has played in the strain variation observed for common STDs such as gonorrhea and chlamydia (Berglund et al. 2002) is not clear and will depend on the amount of contact that takes place between behaviorally distinct groups.

We expect the qualitative prediction that strains specialize to attack specific behavioral subgroups to be testable. In the case of STDs, much of the work of strain typing has been carried out for HIV, which is, of course, a far more complicated infection than can be described by the simple model shown here (Burke and McCutchan 1997; Hu et al. 1998). However, the observed patchy distribution of strains, with different strains dominating in different communities, displays the expected result; in particular, when communities are close knit (such as those associated with injecting drug use), we would anticipate the identity of the dominant viral strain to depend on the level of interaction within the group. A closer look at chlamydia or gonorrhea strains, combined with behavioral data, would be expected to demonstrate similar results.

Several areas of investigation are needed to clarify the impact of social behavior and mixing patterns on pathogen evolution. Specifically, a more detailed knowledge of the properties of currently circulating strains is needed to determine both the extent to which strains can be characterized in the simplistic ways used here and the stability of the characteristics of pathogen strains within chains of transmission. We would like to know the degree to which pathogen strains are clustered within recognized social groups and the coherency of these groups within networks. This will require strain typing of infected individuals as well as observation of the progress of infections within the individual and measurement of mixing behavior. The activities and infrastructure of genito-urinary medicine clinics, particularly relating to contact tracing efforts, will be useful to these ends. Various interacting factors-differences in susceptibility, interaction rates, and transmissibility, for instance-are likely to play a part in strain distribution; a great deal of data will be required before all the issues affecting strain competition and evolution are properly understood.
The models presented here are, by their nature, simplifications of the behavior both of pathogens and of their hosts. The cross-immunity of strains assumed in the models used means that no individual can be simultaneously infected with multiple strains. When investigating coexistence, this is a conservative assumption; if both strains can persist within a single individual, then they are more likely to be able to persist within the population. Hence, the limits for coexistence determined here would be extended if cross-immunity were incomplete. There is evidence of coinfection with gonorrhea strains, for instance, which both enhances coexistence and promotes the development of new strains through genetic exchange (Turner and Garnett 2002; Martin et al. 2003).

We have also assumed that recovery leaves an individual fully susceptible again whereas some acquired immunity is seen both in chlamydia and gonorrhea (Brunham et al. 1996; Cohen et al. 2005), although this immunity may be ineffective or transient. However, we may think about families of strains rather than individual strains, such that the large numbers within each family serve to negate the effect of specific immunity. Nevertheless, the conclusions drawn are in many ways independent of the precise details of the model used; the coexistence seen is a result of the complex dependence of the basic reproductive ratio, $R_{0}$, on the behavior of the population. Although acquired immunity has a small effect on $R_{0}$ in network models (Keeling 1999), this does not affect the conclusion that the outcome of strain competition differs in differing regions of the network.

We have treated the host population as strictly serially monogamous. This may not always be appropriate (although concurrent partnerships can be viewed as frequent repeated relationships within a small set of individuals). In some situations parts of the network might be better treated as polygamous. Even so, the discussion regarding partnership turnover rates is anticipated to apply when a small amount of polygamy is included within a broadly monogamous population; for instance, the inclusion of a polygamous core group in an otherwise monogamous population would result in broadly similar patterns of coexistence. Indeed, polygamous behavior in the core group would further favor fast strains because it removes the necessity to persist in between partnerships whereas areas of slow partnership turnover will continue to favor strains with slower dynamics. Hence, core group polygamy would be expected to extend the parameter region in which coexistence could occur.

The conclusions drawn have resulted from heterogeneities in the population and, in particular, variation in mixing behavior. The core-periphery population structure used here has its closest analogue in the field of STDs but may also be more widely applicable. Instead of thinking 
about sexual partnership turnover, we may consider the turnover of social interactions and contrast regions with different numbers and lengths of interactions, for instance, rural and urban settings. Areas with slow social dynamics place similar restrictions on the spread of infection to the noncore groups in sexual partnership models. It may therefore be the case that varying timescales of interactions should be considered more widely in an effort to understand pathogen strain behavior.

\section{Acknowledgments}

This research was supported by Emmanuel College, Cambridge, and Engineering and Physical Sciences Research Council (K.T.D.E.) and by the Royal Society, National Institutes of Health, and the Leverhulme Trust (M.J.K.). We would like to thank the referees for their considered and constructive comments and suggestions.

\section{Literature Cited}

Altmann, M. 1995. Susceptible-infected-removed epidemic models with dynamic partnerships. Journal of Mathematical Biology 33: 661-675.

Amarasekare, P., M. F. Hoopes, N. Mouquet, and M. Holyoak. 2004. Mechanisms of coexistence in competitive metacommunities. American Naturalist 164:310-326.

Anderson, R. M., and R. M. May. 1992. Infectious diseases of humans. Oxford University Press, Oxford.

Baba, T., F. Takeuchi, M. Kuroda, H. Yuzawa, K. Aoki, A. Oguchi, Y. Nagai, et al. 2002. Genome and virulence determinants of high virulence community-acquired MRSA. Lancet 359:1819-1827.

Bearman, P. S., J. Moody, and K. Stovel. 2004. Chains of affection: the structure of adolescent romantic and sexual networks. American Journal of Sociology 110:44-91.

Berglund, T., M. Unemo, P. Olsen, J. Giesecke, and H. Fredlund. 2002. One year of Neisseria gonorrhoea isolates in Sweden: the prevalence study of antibiotic susceptibility shows relation to the geographic area of exposure. International Journal of STD and AIDS 13:109-114.

Bolker, B. M. 1999. Analytic models for the patchy spread of plant disease. Bulletin of Mathematical Biology 61:849-874.

Bolker, B. M., and S. W. Pacala. 1999. Spatial moment equations for plant competition: understanding spatial strategies and the advantages of short dispersal. American Naturalist 153:575-602.

Boots, M., and A. Sasaki. 1999. "Small worlds" and the evolution of virulence: infection occurs locally and at a distance. Proceedings of the Royal Society of London B 266:1933-1938.

- 2001. Parasite-driven extinction in spatially explicit hostparasite systems. American Naturalist 159:706-713.

Bremermann, H. J., and H. R. Thieme. 1989. A competitive exclusion principle for pathogen virulence. Journal of Mathematical Biology 27:179-190.

Brunham, R. C., J. Kimani, J. Bwayo, G. Maitha, I. Maclean, C. L. Yang, C. X. Shen, et al. 1996. The epidemiology of Chlamydia trachomatis within a sexually transmitted diseases core group. Journal of Infectious Diseases 173:950-956.

Burke, D. S., and F. E. McCutchan. 1997. Global distribution of human immunodeficiency virus-1 clades. Pages 119-126 in V. T. DeVita, S. Hellman, and S. A. Rosenberg, eds. AIDS: etiology, diagnosis, treatment, and prevention. Lippincott-Raven, Philadelphia.

Chesson, P. 2000. Mechanisms of maintenance of species diversity. Annual Review of Ecology and Systematics 31:343-366.

Cohen, C. R., K. M. Koochesfahani, A. S. Meier, C. X. Shen, K. Karunakaran, B. Ondondo, T. Kinyari, N. R. Mugo, R. Nguti, and R. C. Brunham. 2005. Immunoepidemiologic profile of Chlamydia trachomatis infection: importance of heat-shock protein 60 and interferon-gamma. Journal of Infectious Diseases 192:591-599.

Dieckmann, U., and R. Law. 2000. Relaxation projections and the method of moments. Pages 412-455 in U. Dieckmann, R. Law, and J. A. J. Metz, eds. The geometry of ecological interactions. Cambridge University Press, Cambridge.

Dietz, K., and K. P Hadeler. 1988. Epidemiological models for sexually transmitted diseases. Journal of Mathematical Biology 26:125.

Eames, K. T. D., and M. J. Keeling. 2002. Modeling dynamic and network heterogeneities in the spread of sexually transmitted disease. Proceedings of the National Academy of Sciences of the USA 99:13330-13335.

- 2004. Monogamous networks and the spread of sexually transmitted diseases. Mathematical Biosciences 189:115-130.

Ferguson, N. M., and G. P. Garnett. 2000. More realistic models of STD transmission dynamics: sexual partnership networks, pair models and moment closure. Sexually Transmitted Diseases 27: 600-609.

Ferguson, N. M., C. A. Donnelly, and R. M. Anderson. 2001. The foot-and-mouth epidemic in Great Britain: pattern of spread and impact of interventions. Science 292:1155-1160.

Filipe, J. A. N., and G. J. Gibson. 2001. Comparing approximations in spatio-temporal models for epidemics with local spread. Bulletin of Mathematical Biology 63:603-624.

Frabasile, S., A. Delfraro, L. Facal, C. Videla, M. Galiano, S. J. de Sierra, D. Ruchansky, et al. 2003. Antigenic and genetic variability of human respiratory syncytial viruses (group A) isolated in Uruguay and Argentina: 1993-2001. Journal of Medical Virology 71: 305-312.

Frank, S. A. 1996. Models of parasite virulence. Quarterly Review of Biology 71:37-78.

Gibson, M. A., and J. Bruck. 2000. Efficient exact stochastic simulation of chemical systems with many species and many channels. Journal of Physical Chemistry 104:1876-1889.

Gillespie, D. T. 1977. Exact stochastic simulation of coupled chemical reactions. Journal of Physical Chemistry 81:2340-2361.

Grenfell, B. T., O. G. Pybus, J. R. Gog, J. L. N. Wood, J. M. Daly, J. A. Mumford, and E. C. Holmes. 2004. Unifying the epidemiological and evolutionary dynamics of pathogens. Science 303:327332.

Hethcote, H. W., and J. A. Yorke. 1984. Gonorrhea transmission dynamics and control. Vol. 56. Lecture notes in biomathematics. Springer, Berlin.

Hu, D. J., T. J. Dondero, T. D. Mastro, and H. D. Gayle. 1998. Global and molecular epidemiology of HIV. Pages $27-40$ in G. P. Wormser, ed. AIDS and other manifestations of HIV infection. LippincottRaven, Philadelphia.

Johnson, A. M., J. Wadsworth, K. Wellings, and J. Field. 1994. Sexual attitudes and lifestyles. Blackwell Scientific, Oxford.

Johnson, A. M., C. H. Mercer, B. Erens, A. J. Copas, S. McManus, 
K. Wellings, K. A. Fenton, et al. 2001. Sexual behavior in Britain: partnerships, practices, and HIV risk behaviors. Lancet 358:18351842.

Jolly, A. M., and J. L. Wylie. 2002. Gonorrhoea and chlamydia core groups and sexual networks in Manitoba. Sexually Transmitted Infections 78(suppl.):i145-i151.

Keeling, M. J. 1999. The effects of local spatial structure on epidemiological invasions. Proceedings of the Royal Society of London B 266:859-867.

Keeling, M. J., D. A. Rand, and A. J. Morris. 1997. Correlation models for childhood epidemics. Proceedings of the Royal Society of London B 264:1149-1156.

Keeling, M. J., F. M. Jiggins, and J. M. Read. 2003. The invasion and coexistence of competing Wolbachia strains. Heredity 91:382-388.

Kretzschmar, M., and K. Dietz. 1998. The effect of pair formation and variable infectivity on the spread of an infection without recovery. Mathematical Biosciences 148:83-113.

Martin, I. M. C., A. Ghani, G. Bell, G. Kinghorn, and C. A. Ison. 2003. Persistence of two genotypes of Neisseria gonorrhoeae during transmission. Journal of Clinical Microbiology 41:5609-5614.

May, R. M., and R. M. Anderson. 1983. Epidemiology and genetics in the coevolution of parasites and hosts. Proceedings of the Royal Society of London B 219:281-313.

Nowak, M. A., and R. M. May. 1994. Superinfection and the evolution of parasite virulence. Proceedings of the Royal Society of London B 255:81-89.

Rand, D. A. 1999. Correlation equations and pair approximations for spatial ecologies. Pages 100-142 in J. M. McGlade, ed. Advanced ecological theory. Blackwell Science, Oxford.

Read, J. M., and M. J. Keeling. 2003. Disease evolution on networks: the role of contact structure. Proceedings of the Royal Society of London B 270:699-708.

Rohani, P., C. J. Green, N. B. Mantilla-Beniers, and B. T. Grenfell. 2003. Ecological interference between fatal diseases. Nature 422: 885-888.

Rothenberg, R. B., J. J. Potterat, and D. E. Woodhouse. 1996. Personal risk taking and the spread of disease: beyond core groups. Journal of Infectious Diseases 174(suppl.):S144-S149.

Satō, K., H. Matsuda, and A. Sasaki. 1994. Pathogen invasion and host extinction in lattice structured populations. Journal of Mathematical Biology 32:251-268.

Smith, D. J., A. S. Lapedes, J. C. de Jong, T. M. Bestebroer, G. F. Rimmelzwaan, A. D. M. E. Osterhaus, and R. A. M. Fouchier. 2004. Mapping the antigenic and genetic evolution of influenza virus. Science 305:371-376.

Snyder, R. E., and P. Chesson. 2004. How the spatial scales of dispersal, competition, and environmental heterogeneity interact to affect coexistence. American Naturalist 164:633-650.

Tilman, D. 1994. Competition and biodiversity in spatially structured habitats. Ecology 75:2-16.

Turner, K. M. E., and G. P. Garnett. 2002 The impact of the phase of an epidemic of sexually transmitted infection on the evolution of the organism. Sexually Transmitted Diseases 78:20-30.

van Baalen, M. 2002. Contact networks and the evolution of virulence. Pages 85-103 in U. Dieckmann, J. A. J. Metz, M. W. Sabelis, and K. Sigmund, eds. Adaptive dynamics of infectious diseases. Cambridge University Press, Cambridge.

van Baalen, M., and M. W. Sabelis. 1995. The dynamics of multiple infection and the evolution of virulence. American Naturalist 146: 881-910.

van Ballegooijen, W. M., and M. C. Boerlijst. 2004. Emergent tradeoffs and selection for outbreak frequency in spatial epidemics. Proceedings of the National Academy of Sciences of the USA 101: 18246-18250.

Wasserman, S., and K. Faust. 1994. Social network analysis. Cambridge University Press, Cambridge.

Zenilman, J. M., N. Ellish, A. Fresia, and G. Glass. 1999. The geography of sexual partnerships in Baltimore: applications of core theory dynamics using a geographic information system. Sexually Transmitted Diseases 26:75-81.

Associate Editor: Benjamin M. Bolker Editor: Donald L. DeAngelis 